\title{
Segregation by color and stereoscopic depth in three-dimensional visual space
}

\author{
ALBERT W. CHAU \\ University of Hong Kong, Hong Kong \\ and \\ YEI-YU YEH \\ National Taiwan University, Taipei, Taiwan
}

\begin{abstract}
Two experiments were conducted to investigate how color and stereoscopic depth information are used to segregate objects for visual search in three-dimensional (3-D) visual space. Eight observers were asked to indicate the alphanumeric category (letter or digit) of the target which had its unique color and unique depth plane. In Experiment 1, distractors sharing a common depth plane or a common color appeared in spatial contiguity in the $x y$ plane. The results suggest that visual search for the target involves examination of kernels formed by homogeneous items sharing the same color and depth. In Experiment 2, the $x y$ contiguity of distractors sharing a common color or a common depth plane was varied. The results showed that when target-distractor distinction becomes more difficult on one dimension, the other dimension becomes more important in performing visual search, as indicated by a larger effect on search time. This suggests that observers can make optimal use of the information available. Finally, color had a larger effect on search time than did stereoscopic depth. Overall, the results support models of visual processing which maintain that perceptual segregation and selective attention are determined by similarity among objects in 3-D visual space on both spatial and nonspatial stimulus dimensions.
\end{abstract}

An important function performed by the visual system in processing visual information is to select information from the visual scene which is relevant to the current behavior. To achieve this, the visual system has to segregate stimuli in visual space so that parts belonging to the same whole can be linked together and boundaries can be drawn between different wholes. It has been shown that the visual system uses information such as spatial location (see, e.g., Eriksen \& Eriksen, 1974; LaBerge \& Brown, 1986), perceptual organization (e.g., Banks \& Prinzmetal, 1976; Baylis \& Driver, 1992; Driver \& Baylis, 1989), and feature similarity (e.g., Duncan \& Humphreys, 1989; Kramer, Tham, \& Yeh, 1991) in order to achieve segregation. The purpose of the present study is to extend previous work on segregation in two-dimensional (2-D) space and investigate how observers use stereoscopic depth and color information to segregate objects and search for a target in three-dimensional (3-D) visual space.

Humphreys (1981) summarized two models of how multidimensional ${ }^{1}$ stimuli are processed. The first is the independent dimensional processing model, which assumes that some dimensions can be analyzed indepen-

The authors thank Arthur F, Kramer, George J. Andersen, Marc Carter, and an anonymous reviewer for their invaluable comments on an earlier draft of this manuscript. Correspondence concerning this article should be sent to A. W. Chau, Department of Psychology, University of Hong Kong, Pokfulam Road, Hong Kong (e-mail: awlchau $(a)$ hkucc.hku.hk). dently and in parallel. A stimulus is processed on the basis of the first relevant dimension that is resolved. Garner (1974) labeled these dimensions "separable," and it is easy to limit processing to one dimension while ignoring the other. In the framework of information integration (Anderson, 1974), this limitation of processing to one dimension is described as the selection rule, which postulates that observers respond only to information represented in one dimension and ignore the rest; the neglected dimension(s) does (do) not have any effect on the observer's performance.

The second model is the holistic processing model, which suggests that objects are represented by unitary configurations in a multidimensional psychological space. Selective processing of objects in this space depends on the discriminability (or spacing) among them in the psychological space. Because the coordinates of the point representing an object in the psychological space are the values that the object assumes on the individual dimensions, all dimensions jointly determine the selective processing of objects. In the information integration perspective, an additive or a multiplicative rule is followed.

It has been shown that differences in stereoscopic depth can be used for boundary segmentation in visual space or for shape recognition (see, e.g., Cavanagh, 1987; Julesz, 1971). Nakayama and Silverman (1986a, 1986b) have shown that stereoscopic depth is the primary dimension for segregation in 3-D visual space. Their subjects were able to search for targets defined by stereoscopic depth and each of the following dimensions in a 
parallel manner: motion (up vs. down), color (red vs. green), polarity (black vs. white), orientation (horizontal vs. vertical) or size (large vs. small) - as indicated by a slope of less than $12 \mathrm{msec}$ per item in the linear function between search time and number of distractor items. Nakayama and Silverman (1986b) postulated that "a parallel search can be done within each depth plane (in turn) without interference from target-like distractors in another depth plane" (p. 265). They further proposed that "visual features like color and motion could be separately encoded and duplicated at other disparities" (p. 265).

Some other studies have refuted the idea of independent processing for objects at different depths. Drobnis and Lawson (1976) found that the Poggendorf illusion existed even when the bisecting rectangle and the oblique line were in different depth planes. Lehmkuhle and Fox (1980) used random dot stereograms to present a target that was a Landolt $\mathrm{C}$ with the gap position randomly varied, and a mask that was an annulus that surrounded the target. The magnitude of the lateral masking effect was assessed by the observer's accuracy in reporting the location of the gap. It was found that the lateral masking effect diminished as the target and mask became more separated in depth. Findings that demonstrated interference between depth planes were also obtained by Fox and Patterson (1981), Andersen (1990), and Andersen and Kramer (1993).

The visual search paradigm has shown the usefulness of color as a dimension for distinguishing a target from distractors. E. C. Carter and R. C. Carter (1981) showed that the color difference between a target and distractors determined the conspicuousness of the target, as measured by relative fixation rate and reaction time. Following the same line, R. C. Carter (1982) found that the slope of the search time function increased as the target and distractors became more similar in color. Using a target identification task, Baylis and Driver (1992) found that distant distractors that shared the same color with the target caused more interference to the target than did near distractors that were colored differently.

The spatial organization and number of color groups in the display also affect how visual search is conducted. Bundesen and Pedersen (1983) presented displays in which same-color distractors appeared either in spatial proximity (organized displays) or at randomly assigned locations (scrambled displays). The task was to identify the alphanumeric category (letter or digit) of the target, which was defined by its unique color. Identification time increased linearly with the number of colors in the organized displays, and with the number of phenomenally perceived separate color groups in the scrambled displays. Such findings indicated that items in visual space are segregated by color and that observers examine groups of same-color items in the search for the uniquely colored target.

Humphreys (1981) studied the relative usefulness of color and location information when they were redundantly presented. His results indicated that color infor- mation was first used to parse the target from the distractors. When color was informative in the sense that it consistently specified the target, only color information was used to identify the target. When color did not reliably specify the target, observers performed a location check to determine the shapes of the distractors. Using a response-deadline technique in his third experiment, he found that color information was resolved more quickly than location information. Observers resorted to location information only when color was not informative. These results supported a selection rule based on color when both color and location information are available.

In this study, we examined whether the preceding findings obtained with 2-D displays could be obtained with 3-D displays. The question of interest was how color and stereoscopic depth information might be used for perceptual segregation and target search.

For the selection rule to be followed, stimulus attributes must be separately processed and represented in such a way that parallel processing can occur. Furthermore, the processing in the separate mechanisms should be independent, so that processing in one mechanism has little influence on the processing in the other mechanism. The issue of whether color and binocular disparity are processed independently has been a controversy in both psychophysical and physiological research. It has been demonstrated that depth is not perceivable in random dot stereograms that are isoluminant (Gregory, 1977; Lu \& Fender, 1972) or have certain dot-to-background luminance ratios (de Weert, 1979). Livingstone and Hubel (1988) proposed that color and form information are processed in the parvocellular pathway, whereas luminance, motion, and binocular disparity information are processed in the magnocellular pathway. These studies suggested that only achromatic, not chromatic, signals have access to the cortical mechanisms that are responsible for stereopsis.

On the other hand, some studies have shown that the color-opponent channel is indeed involved in the processing of binocular disparity information: chromatic cues are useful in disambiguating ambiguous wallpaper stereograms (Jordan, Geisler, \& Bovik, 1990), perception of stereo movement (Tyler \& Cavanagh, 1991), and segregation of overlapping transparent surfaces in random dot stereograms (Akerstrom \& Todd, 1988). Schiller, Logothetis, and Charles (1990) showed that lesion of the parvocellular lateral geniculate nucleus in rhesus monkeys impaired both color discrimination and fine stereopsis. These results suggest that the parvocellular and magnocellular mechanisms are not independent.

Given that both color and stereoscopic depth are powerful cues for segregation on their own and that there is evidence that they could be processed independently, it is possible that a selection rule, or just one dimension, is used for perceptual segregation and target search. On the contrary, the holistic processing model predicts that stereoscopic depth and color should jointly determine how the visual system segregates objects in visual space. This view is also consistent with the object-based mod- 
els of selective attention which maintain that the initial preattentive stage parses items in the visual field into perceptual units or groups according to certain principles of perceptual organization (Baylis \& Driver, 1992; Duncan, 1984; Harms \& Bundesen, 1983; Prinzmetal, 1981), as well as with models that emphasize targetdistractor similarity and distractor-distractor similarity (e.g., Duncan \& Humphreys, 1989).

The task in the two experiments reported here was to identify the alphanumeric category (letter or digit) of the target that was distinguished redundantly from the distractors by its unique color and unique stereoscopic depth. Because the target was redundantly defined by the two dimensions, observers could use color and/or stereoscopic depth to search for the target. In Experiment 1, we examined whether observers segregate objects by information on only one or both dimensions. In Experiment 2, we studied the importance of display organization in the $x y$ plane and tested the hypothesis that observers make differential use of the two dimensions to search for the target if information on one dimension is less effective.

\section{EXPERIMENT 1}

The objective of this experiment was to reveal how information of color and stereoscopic depth is used to segregate stimuli and perform visual search in 3 -D visual space. The distractors were organized into various color clusters and depth planes so that distractors sharing the same color or stereoscopic depth appeared in a spatially contiguous region in the $x y$ plane. This arrangement was adopted to maximize the likelihood that homogeneous distractors (distractors of the same color and same depth) would be segregated into the same unit. Display set size, number of distractor colors, and number of distractor depth planes were manipulated orthogonally. Two control conditions were included as well. In the color-only condition, all stimuli were presented in the same depth plane so that observers only had color information to identify the target. In the depth-only condition, the whole display was in one color and the target could only be identified by its unique depth plane.

If observers adopt a selection rule and merely use color to segregate objects and search for the target, the number of distractor colors should be the only factor that affects search performance, and the effect should be similar to that in the color-only condition. Likewise, if segregation and target search are based solely on stereoscopic depth, only the number of distractor depth planes should influence search time, and the effect should be similar to that in the depth-only condition. On the other hand, if both dimensions are needed to perform perceptual segregation, both the number of distractor colors and the number of distractor planes should affect search time.

\section{Method}

Observers. Six graduate and two upperclass undergraduate psychology students at the University of Wisconsin-Madison served as observers in both experiments. They received $\$ 4.50$ per hour of their participation.

Each observer's vision was tested with an Optec 2000 vision tester. They all passed vision tests on near and far visual acuity (20/20 Snellen equivalent or better), stereoacuity (27" of arc or better), near and far phoria (less than or equal 0.5 prism diopters of deviation), near lateral phoria (between 0.0 and -6.0 prism diopters), far lateral phoria (between 2.33 and -1.66 prism diopters), and color vision. They also passed a color vision test using the standard pseudoisochromatic plates provided by Igakushoin.

Apparatus and Stimuli. An IBM-compatible 386 personal computer was used for data collection and cont rol of stimulus presentation. The stimuli were presented on a Tektronix SGS 625 stereoscopic display system, which was coupled to the 386 with a Tektronix Stereoscopic Graphics Adapter Card SGS 100. Observers wore a pair of crossed Polaroid filters to obtain dioptic separation for depth perception (see Yeh \& Silverstein, 1990, for a detailed discussion of the apparatus). When the disparity of an item assumed a nonzero value, two separate images were generated on the screen and each of them was seen by a different eye. In the present studies, we used negative values of disparity to indicate crossed disparity at which the items were perceived as situated in front of the screen, and positive values for uncrossed disparity at which the items appeared to be behind the screen. The fixation plane was always on the screen that had zero disparity.

A chinrest was used to maintain the viewing distance at $152 \mathrm{~cm}$. The stereoscopic display monitor was situated at the end of a dark viewing tunnel, and the bezel of the monitor was covered with black cloth to make it invisible in the tunnel. The room was dimly lighted when the experiment was in progress

Each display consisted of a number of alphanumeric characters (letters or digits) occupying a subset of locations in an imaginary $8 \times 8$ matrix. Seven digits $(0,5$, and 8 excluded $)$ and 19 consonants ( $B$ and $S$ excluded) served as stimulus items. Digits and letters in the display were randomly chosen from the corresponding set with replacement. At a viewing distance of $152 \mathrm{~cm}$, the matrix subtended $6^{\circ} \times 6.2^{\circ}$ and each element subtended $7.8^{\prime} \times 10^{\prime}$ of arc. Adjacent columns or rows were separated by $42^{\prime}$.

All display items were presented in a bluish gray background (CIE coordinates, $x=0.2895, y=0.2772$ : luminance $=0.292$ $\mathrm{cd} / \mathrm{m}^{2}$ ). Five colors were used for the display items and their coordinates in the CIE color space: red $(0.6241,0.3410)$, green $(0.2738,0.6004)$, magenta $(0.3076,0.1650)$. cyan $(0.1804,0.2079)$ and yellow $(0.4490,0.4707)$. These colors were chosen in order to achieve a high degree of discriminability. The five disparities used in the experiment were: $-20^{\prime},-10^{\prime}, 0^{\prime}, 10^{\prime}$, and $20^{\prime}$ of are.

Subjective brightnesses of the colors were matched for each observer prior to the experiment by using the minimum border method (Wagner \& Boynton, 1972). In this task, a reference white square $(\mathrm{CIE}$ coordinates, $x=0.3479, y=0.3365$; luminance $=$ $3.0 \mathrm{~cd} / \mathrm{m}^{2}$ ) and a test square in the color to be matched were shown side by side sharing a common border on the screen. Both squares measured $25^{\prime} \times 25^{\prime}$ of arc. At the beginning of each trial, the test square was shown at a luminance that was determined randomly. The observer then used a mouse to adjust the luminance of the test square in order to minimize the common border between the squares. Each observer was given a block of 10 replications for each color; and the mean of the middle eight luminances was used as the luminance for that color in the actual experiment. The order of administering the color blocks was determined randomly for each observer.

An element with a unique color and a unique stereoscopic depth (disparity) served as the target in each display. In each condition, the target was a digit on half of the trials and a letter on the other half of the trials. The five colors and five disparities were used equally frequently for the target. For each target color, each possible combination of the other four colors was used for the distrac- 
tors on equal numbers of trials. For instance, in the condition which had one distractor color, for each target color, each of the four nontarget colors was used as the distractor color equally frequently. In the condition of two distractor colors, there were six equally frequent combinations for the distractor colors $\left(6={ }_{4} C_{2}\right.$; two nontarget colors were chosen from four distractor colors on each trial). In the condition of four dist ractor colors, all four nontarget colors were used in every display. The same arrangement applied to the generation of target and distractor disparities.

The assignment of color and disparity to the distractors began with assigning a color and a disparity to each location in the $8 \times 8$ matrix. Depending on the experimental condition, the matrix was first divided into the number of horizontal and vertical regions required by the trial. Each horizontal region was assigned a distractor color, and each vertical region, a disparity. As a result, the $8 \times 8$ matrix was divided into a number of kernels, each of which consisted of items of a unique combination of color and disparity. All color and disparity assignments were random and without replacement.

Figure 1 shows a sample display which had two distractor colors and two distractor planes. Locations of distractors were randomly picked within each kernel so that an equal number of distractors appeared in each kernel. Half of the distractors in each kernel were digits, while the other half were letters. The target location was randomly selected in the display with the constraint that it did not disturb the continuity within any distractor kernel.
On half of the trials which were chosen randomly, the directions of the color and disparity assignments were swapped so that samecolor items were organized vertically and same-disparity items horizontally.

In the color-only condition, all items were presented at the same depth (disparity) on each trial. The way of assigning colors followed that of the experimental condition so that distractors of the same color formed a kernel. The five disparities used in the experimental condition were employed as the display disparity equally often in a random order across trials. Displays in the depth-only condition were generated similarly.

Design and Procedure. Three within-subjects factors were manipulated in the experiment: display set size $(25,37$, and 49 items), number of distractor colors (one, two, or four) and number of distractor depth planes (one, two, or four). In each of the three sessions of the experimental condition, there were 20 trials for each combination of the three factors, rendering a total of 540 trials. The order of trial presentation was randomized for each session and for each observer. The experimental trials were preceded by 54 practice trials, 2 for each combination of the three factors. The observers were given a short break after the practice trials and after each block of 90 experimental trials. In the first session, the observers also underwent the vision test and the minimum border luminance matching task. The first session lasted about $1.5 \mathrm{~h}$; the other two sessions were about $45 \mathrm{~min}$ long.
Distractor Plane 1

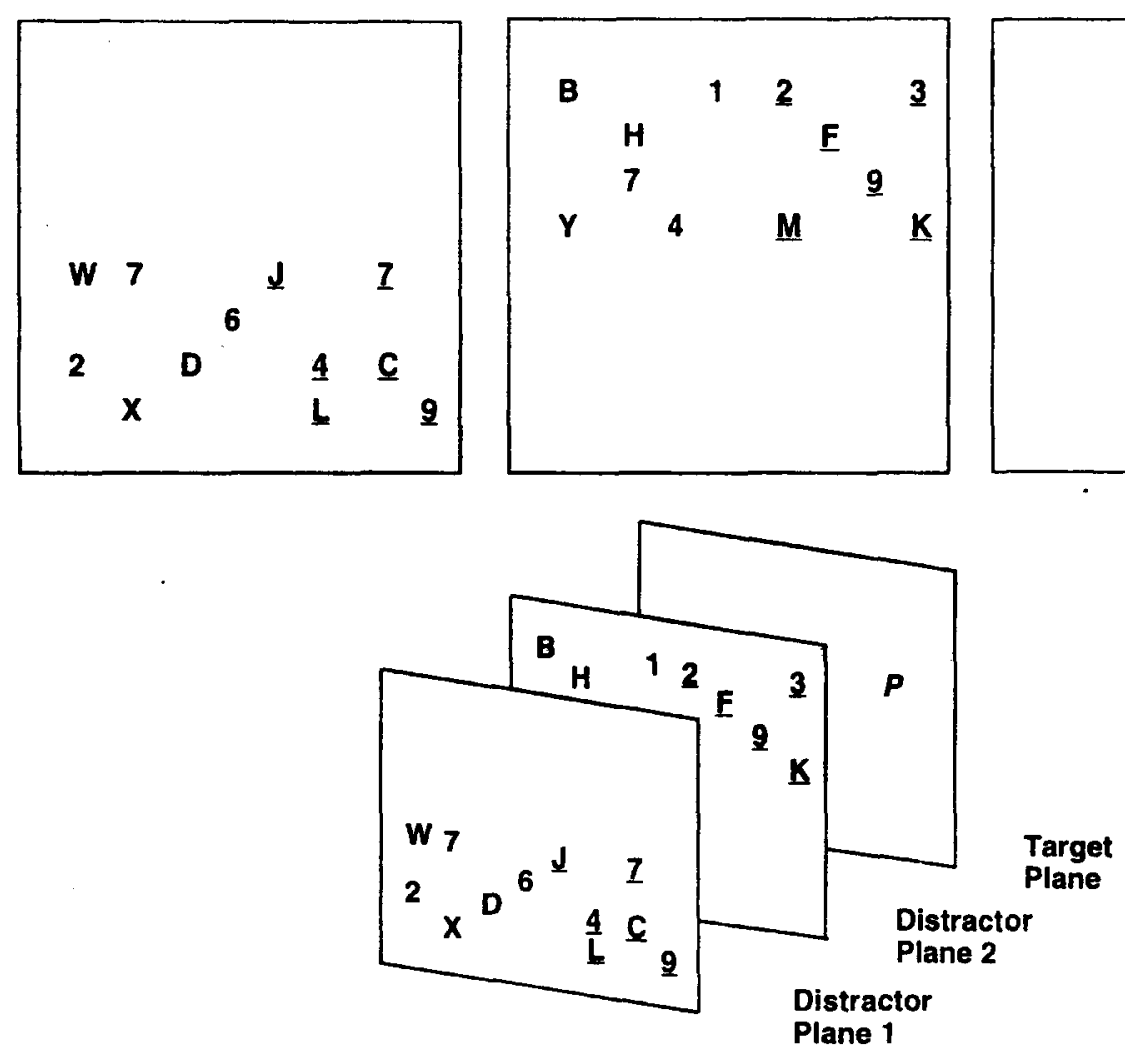

Figure 1. A sample display used in Experiment 1. The display shown here consists of two distractor planes and two distractor colors. The upper panel shows how the planes are constructed. Items with and without underscore represent distractors in two different colors. The target has its own color (shown in a different font). In the actual displays, all items were in the same font and not underscored. The bottom panel shows how the planes were stacked in the 3-D display. On half the trials, the row and column arrangements for color and disparity were reversed so that distractors in the same disparity appeared in the same columns and distractors in the same color appeared in the same rows. 
Two within-subjects factors were manipulated in the color-only control condition: display set size $(25,37$, and 49 items) and number of distractor colors $(1,2$, or 4$)$. Similarly, display set size $(25$, 37 , and 49 items) and number of dist ractor planes (1,2, or 4 ) were manipulated in the depth-only control condition. Each control session consisted of 540 experimental trials (60 trials for each combination of the factors), preceded by 27 practice trials (three for each combination of the factors). All trials were presented in random order. Again the observers received a short break after the practice trials and each block of 90 experimental trials. Each session lasted about $45 \mathrm{~min}$.

Observers completed the experiment in five sessions, with sessions on separate days. The experimental sessions occupied the first 3 days, and the control conditions took up the last 2 , in random order. Each trial began with a fixation cross at the center of the screen. One second later, the fixation cross was replaced by the stimulus array, which stayed on until the observer made a response. The next trial commenced about $500 \mathrm{msec}$ later.

Observers made their responses with a mouse. Half of the observers pressed the left button for a letter target and the right button for a digit target, and the remaining observers had the opposite key assignment. Key assignment for each observer was randomly determined and stayed unchanged throughout both experiments.

\section{Results}

Both search time of correct responses and error rate were analyzed. Outlying search times were removed prior to the main analysis with the following procedure. Means and standard deviations were computed for each cell and for each observer on the basis of the correct responses. Only search times of the correct responses within $2.5 \mathrm{SD}$ s from their respective cell means were included in the analysis. On the average, $1.83,1.76$, and $1.74 \mathrm{ob}-$ servations (out of 60 ) were removed from each cell in the color-only, depth-only, and experimental conditions, respectively. No observer had more than 2.4 observations removed from any ce!l.

All post hoc comparisons reported here were performed with Tukey's (a) test.

Color-only control condition. The mean error rate was $2.63 \%$ across all observers. There was no evidence of speed-accuracy tradeoff, since conditions with longer search times were not associated with lower error rates. The correlation between search time and error rate over the nine experimental conditions was not significant $[r(7)=.220, p>.05]$.

Preliminary analysis of search time showed no main effect or interaction due to type of target. Search times for letter and digit targets were thus pooled for subsequent data analysis. The same was also true for the other two conditions of this experiment and for Experiment 2.

Correct search times were submitted to a $3 \times 3$ (number of distractor colors $X$ display set size) withinsubjects analysis of variance (ANOVA). The main effect of number of distractor colors was significant $[F(2,14)=$ $25.29, p<.05]$. Trend analysis revealed that search time increased linearly with number of distractor colors $[F(1,7)=25.76, p<.05]$. (See Figure 2.) Display set size had no effect on search time. The interaction was not significant either.

Depth-only control condition. The mean error rate was $4.32 \%$. Again there was no evidence of speed- accuracy tradeoff; the slower conditions were associated with higher error rates $[r(7)=.804, p<.05]$.

A $3 \times 3$ (number of distractor planes $\times$ display set size) within-subjects ANOVA revealed a significant main effect of number of distractor depth planes $[F(2,14)=$ $33.84, p<.05]$. Trend analysis showed that search time linearly increased with number of distractor planes $[F(1,7)=37.87, p<.05]$. (See Figure 2.) No effects of display set size were found.

Experimental (color and depth) condition. The mean error rate was $3.06 \%$. As in the previous two conditions, a speed accuracy tradeoff was not found. The correlation between search time and error rate over the 27 experimental conditions was not significant $[r(25)=$ $.143, p>.05]$.

A $3 \times 3 \times 3$ within-subjects ANOVA was performed on search time, with display set size, number of distractor colors, and number of distractor depth planes as factors. There were significant main effects of display set size $[F(2,14)=8.25, p<.05]$, number of distractor colors $[F(2,14)=53.78, p<.05]$, and number of distractor depth planes $[F(2,14)=18.64, p<.05]$. For display set size, the responses for 25 or 37 items were significantly slower than those for 49 items; with mean search times of $1,070,1,064$, and $1,039 \mathrm{msec}$, respectively. The mean search times for one, two, and four distractor colors were $958,1,039$, and $1,177 \mathrm{msec}$, respectively, and they were significantly different from one another. The same was true for number of distractor depth planes, where the mean search times were $973,1,071$, and $1,130 \mathrm{msec}$ for one, two, and four distractor depth planes, respectively.

All two-way and three-way interactions were significant: number of distractor colors $X$ number of distractor depth planes $[F(4,28)=16.32, p<.05]$, display set size $\times$ number of distractor colors $[F(4,28)=4.25, p<.05]$, display set size $\times$ number of distractor depth planes $[F(4,28)=3.82, p<.05]$, and display set size $\times$ number of distractor colors $\times$ number of distractor depth planes $[F(8,56)=2.44, p<.05]$.

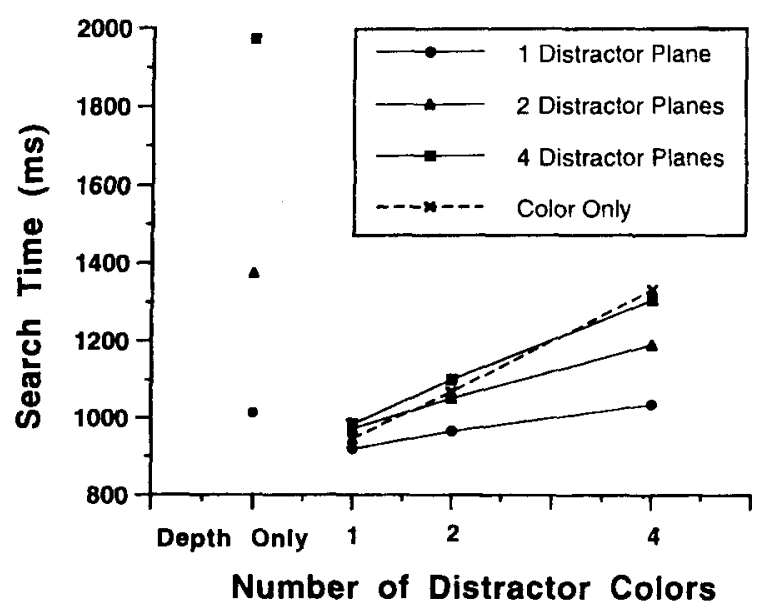

Figure 2. Mean search time as a function of number of distractor colors and number of distractor planes in Experiment 1. 
Post hoc analyses were conducted to examine these interactions further. Figure 2 shows the multiplicative effect of number of distractor colors and number of distractor depth planes on search time. In general, search time increased with number of distractor colors and the rate of increase was more rapid with more distractor depth planes. Trend analysis revealed that the linear by linear trend was the only significant component of the interaction $[F(1,7)=19.89, p<.05]$. For the interaction between display set size and number of distractor colors, search time decreased with increases in display set size when there were four distractor colors but did not vary when only one or two distractor colors were present (Figure 3a). Display set size also had a decreasing effect on search time when there were two or four distractor depth planes (Figure 3b). The three-way interaction is shown in Figure 4. In general, search time decreased more quickly with increases in display set size when the display consisted of more kernels of homogeneous items.

\section{Discussion}

Two major findings were obtained. First, number of distractor colors and number of distractor depth planes together have a multiplicative effect on search time. Second, there was an inverse relationship between search time and display set size. This inverse relationship became more noticeable when there were more kernels of homogeneous items in the display.

The linear fan pattern of search time in Figure 2 supports the hypothesis that color and stereoscopic depth information jointly determine the segregation of 3-D vi- sual space. The significant linear by linear trend of the interaction indicates that the manipulations of number of distractor colors and number of distractor planes had a multiplicative effect on search time. Also, the correlation between search time and the product of number of distractor colors and number of distractor planes was close to unity $[r(7)=.964, p<.05]$. Since the product of these two numbers is the number of kernels in the display that are defined by items of the same color and same depth, the statistic again indicates that visual search for a target jointly defined by stereoscopic depth and color involves an examination of clusters of items that are common in both stereoscopic depth and color. It echoes the results of Bundesen and Pedersen (1983), which suggest that visual search for a target uniquely defined by color proceeds by examining units formed by same-color items. This finding also supports the postulation that stereoscopic depth information is combined with color information in executing certain visual functions such as segregation of objects in visual space. Given that useful information in the real world could be coded by both stereoscopic depth and color (e.g., to look for a red sign $20 \mathrm{ft}$ away), it is to the benefit of the visual system to take advantage of both information sources.

The search strategy of examining kernels of similar items also applies to the control conditions in which we found a significant linear trend between search time and number of homogeneous kernels (same-color items in the color-only condition and same-depth items in the depth-only condition). However, the slope of the linear function between search time and number of kernels dif- (a)

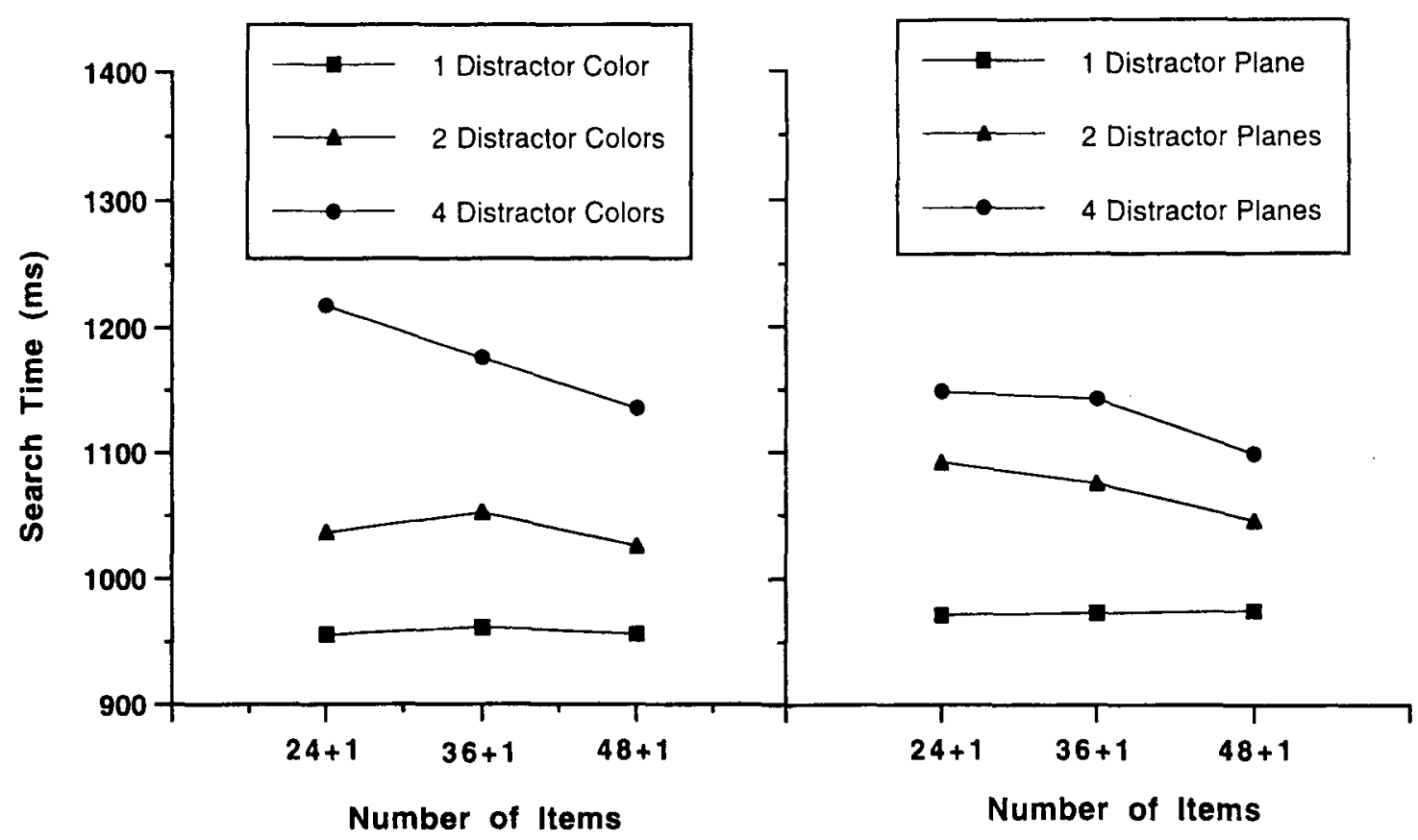

Figure 3. Mean search time as a function of display set size and (a) number of distractor colors and (b) number of distractor planes in Experiment 1. 


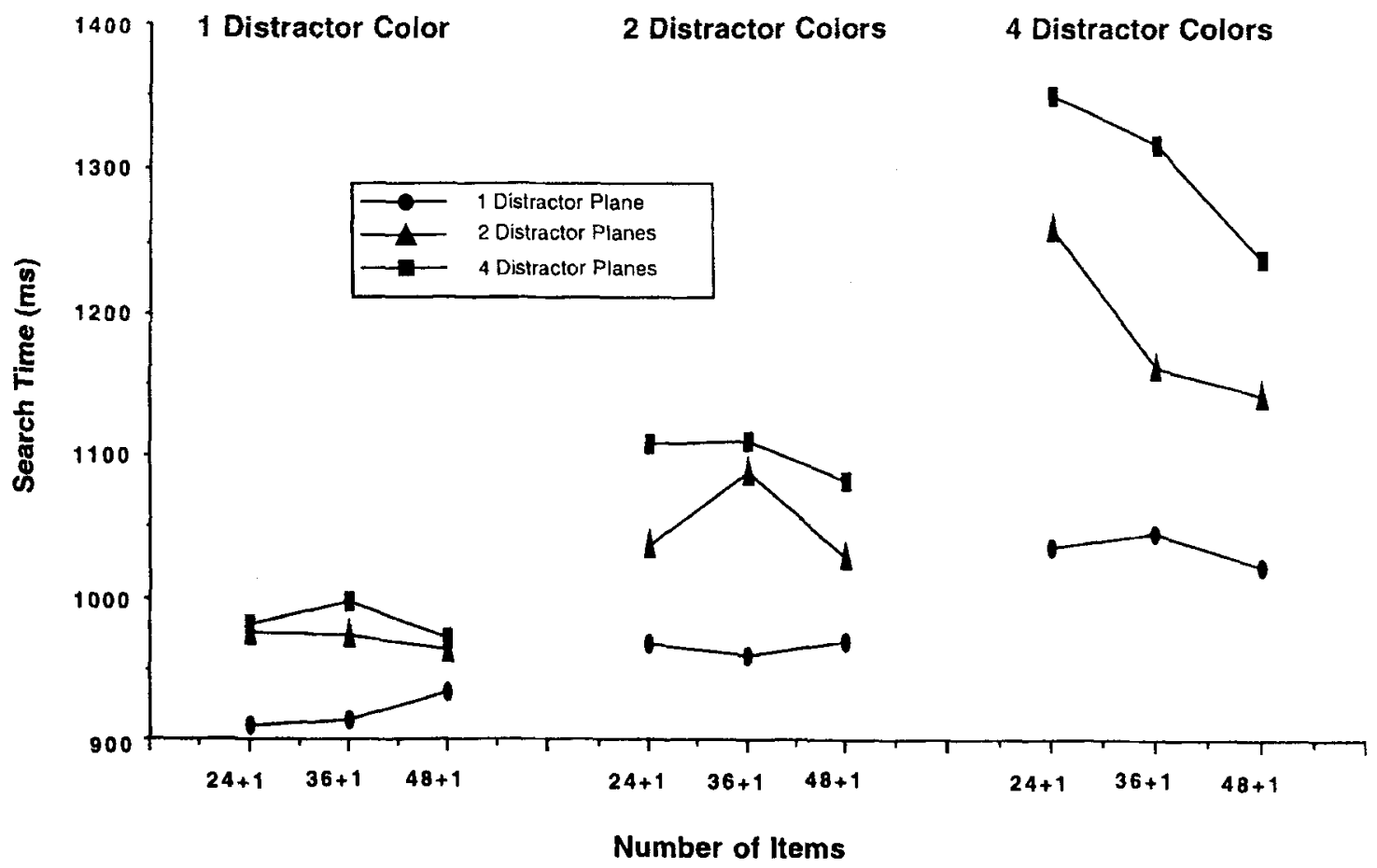

Figure 4. Mean search time as a function of display set size, number of distractor planes, and number of distractor colors in Experiment 1.

fered markedly across the three conditions: 26,129 , and $317 \mathrm{msec}$ per kernel for the experimental, color-only, and depth-only conditions, respectively. The much greater slopes of the control conditions again suggest that visual search for the target is more efficient with two information sources than with one. This benefit of the additional information source is more remarkable if the additional information source clearly specifies the target. For instance, although increasing the number of distractor planes led to enormous search time increments in the depth-only control condition, the effect was much attenuated when the distractors were presented in a different color (the experimental conditions in which there was one distractor color). The observer was able to make efficient use of the additional color information to perform the search.

The preceding findings are also predicted by the theory of Duncan and Humphreys (1989). In their model, similarity among the items in the display plays a dominant role in determining how the items are segregated into various units. Beck, Prazdny, and Rosenfeld (1983) have also provided evidence to show that texture regions are segregated on the basis of a similarity function that depends on features common among and features unique to the objects in a locality. This line of thought can be applied to the present experiment; thus, distractors which are similar in color and depth are parsed into the same structural unit because of the similarity among them. Once the distractors fall into structural units other than that of the target, they are rejected very efficiently be- cause nontarget structural units are denied at the level of preattentive processing.

A comparison between the search time functions of the two control conditions suggests that examination by color seems to be faster than examination by depth. As the slopes indicate, given the manipulations in the present study, an additional distractor color only lengthened the search time by $129 \mathrm{msec}$, whereas an additional distractor plane required $317 \mathrm{msec}$ on the average. We will discuss this point again when we describe Experiment 2.

The finding that search time decreased with increases in display set size can be explained by a number of possible mechanisms. Here the reader is reminded that the size of the full display array is constant. An increase in display set size means a reduction in interitem distance. This reduction results in a better continuity within the distractor kernels and increases the likelihood of detecting a feature gradient between the target and distractors (Sagi \& Julesz, 1987). Both changes tend to strengthen the grouping among the distractors and therefore make the target more distinguishable and detectable (Bacon \& Egeth, 1991). Furthermore, as pointed out by Bravo and Nakayama (1991), mutual inhibition among the distractors involves local computation and should be more effective if the distractors are close together. Therefore, search time should decrease as display set size increases,

Although several other researchers have also reported a negative relationship between search time and display set size (e.g., Bacon \& Egeth, 1991; Bravo \& Nakayama, 1992; Sagi \& Julesz, 1987), this was not the case when 
Bundesen and Pedersen (1983) used a task similar to that in the present experiment. Bundesen and Pedersen hypothesized and found that increasing display set size increased with increases in the density of the display and, as a consequence, lengthened the time to determine the alphanumeric category of the target. The discrepancy in the findings is possibly due to the differences in the stimulus configurations used. Bundesen and Pedersen used items of $0.69^{\circ} \times 1.04^{\circ}$ in size and an interlocation separation of $1.21^{\circ}$ both horizontally and vertically. The ratios between interlocation separation and item size were 1.75 horizontally and 1.16 vertically. In the present study, the items measured $0.13^{\circ} \times 0.17^{\circ}$, with both horizontal and vertical separation at $0.7^{\circ}$. The interlocation separation to item size ratios were 5.4 horizontally and 4.1 vertically. It can be seen from the ratios that the items in the present display were more scattered than those in Bundesen and Pedersen's study. The difference in the "density" of the display becomes more acute when one compares the display set sizes with the size of the matrix from which the distractor locations were chosen. Bundesen and Pedersen (1983) presented 8, 16, or 24 items in a $5 \times 5$ matrix; 25,37 , or 49 items were displayed in an $8 \times 8$ matrix in the present study. A visual inspection of the exemplar display in their article reveals that their displays were much more densely packed than ours.

The current stimulus configuration is more comparable to the one used by Bacon and Egeth (1991), who also found a negative relationship between search time and display set size. The width and height of their items, as well as the horizontal and vertical separations between adjacent locations, all measured $0.67^{\circ}$. Though their interlocation separation to item size ratio (1.00) was much smaller than that in the present study, they presented only $4,8,16$, or 32 items in a $10 \times 10$ matrix. A visual inspection of the sample displays in their article suggests that the items in their displays were also rather scattered.

The preceding comparisons suggest that adding distractors can reduce search time if the additional distractors enhance grouping among the distractors. In Bundesen and Pedersen's (1983) study, the distractors were so close to one another that they were grouped rather effectively even in the smallest display. Therefore, adding distractors did not improve grouping much and hence did not reduce search time. On the other hand, the displays in the present study and in Bacon and Egeth (1991) were rather loosely packed, so that adding distractors resulted in better grouping among the distractors. Consequently, search time was reduced.

The interactions between display set size and the other two factors in the experiment indicate that the negative relationship between search time and display set size is more noticeable when more kernels are formed by increasing the number of dist ractor colors and/or the number of distractor depth planes (Figures 3 and 4). To account for such findings, it should be noted that the distractors in the small display sets were more scattered within the kernels defined by distractor color and dis- tractor depth than those in the large display sets. For instance, with a display showing 25 items and four distractor colors, only 6 of the 16 locations in each color region were occupied by distractors. This lack of spatial proximity and continuity among the distractors hampers the effectiveness of grouping them into the same unit. When the display set size was enlarged to 49 in the preceding example, the number of distractors in each color region increased to 12 . As discussed above, this augmentation in distractor density facilitates the formation of perceptual groups among the distractors and hence reduces search time. The effect of adding distractors was not so obvious when there were only a few kernels in the display (e.g., in the control conditions, in which there were only 1,2 , or 4 regions; or in the experimental condition of two distractor colors and one distractor plane, in which only two kernels existed). In such displays, more distractors were present in each region and they grouped well even when the display set was small. Hence, enlarging the display set did not lead to much better grouping of distractors or much reduction in search time.

In summary, the results of Experiment 1 support the hypothesis that both color and stereoscopic depth are used to segregate items in 3-D visual space, and observers searched for the target by examining the distinctive kernels formed by items of the same color and the same stereoscopic depth.

\section{EXPERIMENT 2}

In Experiment 1, the stimulus array was designed to enhance grouping of items by stereoscopic depth and/or color. Items of the same color or same depth were allocated to contiguous kernels in the $x y$ plane. In this experiment, we examined the importance of spatial proximity in perceptual segregation and studied the relative use of the two dimensions when the target and distractors intermingled with each other in the $x y$ plane.

The importance of spatial proximity for perceptual grouping has been well documented, first by Gestalt psychologists as the law of proximity, and later by contemporary psychologists (e.g., Coren \& Girgus, 1980; Duncan, 1984). When distractors sharing the same attribute are located near one another, they are more likely to be segregated into the same perceptual unit by the principles of similarity and proximity grouping. In contrast, when such homogeneous distractors become less organized in their spatial locations, two factors contribute to hinder their grouping. First, spatial proximity would favor grouping of distractors that are spatially close together even though they may be distinct on some dimension. Second, the lack of good continuation among the homogenous distractors also precludes effective grouping; consequently, the perceptual units will be smaller in size and greater in number. Therefore, if the distractors similar on a certain dimension become more disorganized in the $x y$ plane, it is more difficult to search for the target by examining the units formed on the basis 
of this dimension. In other words, the less organized dimension will become a less effective dimension for visual search, and the other dimension will become more important and should have a larger effect on search time.

In Experiment 2A, display set size, number of distractor planes, and organization of items by color were manipulated. The last variable consisted of four different levels which ranged from the continuous condition, in which same color distractors appeared in spatial proximity in the $x y$ plane, to the scrambled condition, in which color was randomly assigned to distractors at different locations. If stereoscopic depth information becomes more useful in visual search as color organization decreases, search time should increase more rapidly with increases in the number of distractor planes as color organization changes from continuous to scrambled. In Experiment 2B, organization of items by stereoscopic depth was manipulated in the same manner as was color organization in Experiment 2A. Following the above argument, when items of the same depth are less organized in the $x y$ plane, it is more difficult for observers to use stereoscopic depth information to search for the target among the distractors. Therefore, search time is expected to increase more rapidly with increases in number of distractor colors when depth organization diminishes.

\section{Method: Experiment 2A}

Apparatus and Stimuli. The apparatus and stimuli were identical to those in Experiment 1 except for two modifications in the construction of the stimulus array. First, all five colors used in Experiment 1 were used on every trial (one for the target and four for the distractors). Second, distractor colors were assigned to the locations in the $8 \times 8$ array in accordance with the level of the color organization variable.

Design and Procedure. There were three within-subjects factors. The first two were display set size $(25,37$, or 49 items $)$ and number of distractor depth planes (one, two, or four). Four dis- tractor colors were included on every trial. Organization of distractor colors, the third independent variable, had the following four levels:

Continuous. Distractors that shared the same color fell into contiguous horizontal regions in the $x y$ plane. The display was similar to the one in Experiment 1 which had four distractor colors (Figure 5a).

Small separation. The $8 \times 8$ matrix was first divided into sixteen $2 \times 2$ submatrices. Distractors of the same color were assigned to submatrices that were diagonally next to each other (Figure $5 b$ ).

Large separation. As in the previous condition, the $8 \times 8$ matrix was first divided into sixteen $2 \times 2$ matrices as above. Distractors of the same color were assigned to submatrices that were separated by one row of submatrices of other colors (Figure $5 \mathrm{c}$ ).

Scrambled. Distractor colors were randomly assigned to the locations in the $8 \times 8$ matrix, with each distractor color assigned to an equal number of locations.

For all four levels of color organization, same-disparity items were assigned to spatially contiguous regions in the $x y$ plane so that items within a column of submatrices were in the same depth plane. On half of the trials that were selected randomly, the directions of color and disparity assignments were switched so that items of the same disparity appeared in the same row of submatrices and the manipulation of color organization was applied to the columns.

Each observer underwent three 1-h sessions, each one on a separate day. In each session, the observer first received 72 practice trials, 2 for each combination of the experimental factors, followed by 720 experimental trials, 20 for each experimental condition. The observers were allowed a short break after the practice trials and after each block of 90 trials. The presentation of trials was similar to that in Experiment 1.

\section{Results}

Extreme search times were removed with the same procedure as in Experiment 1. On the average, $1.78 \mathrm{ob}-$ servations (out of 60 ) were deleted from each cell. No observer had more than 2 observations eliminated from any cell. (a) Continuous

\begin{tabular}{|c|c|c|c|}
\hline Red & Fed & Red & Red \\
\hline Magenta & Magenta & Magenta & Magenta \\
\hline Cyan & Cyan & Cyan & Cyan \\
\hline Green & Green & Green & Green \\
\hline
\end{tabular}

(b) Small Separation

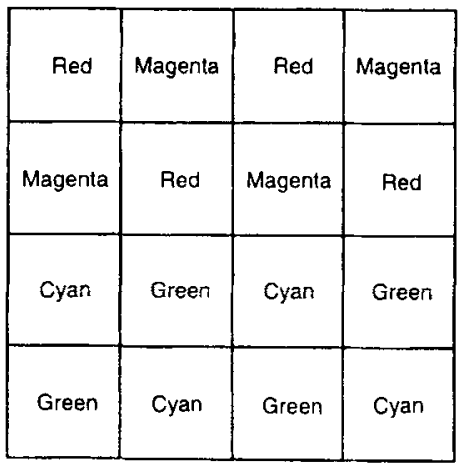

(c) Large Separation

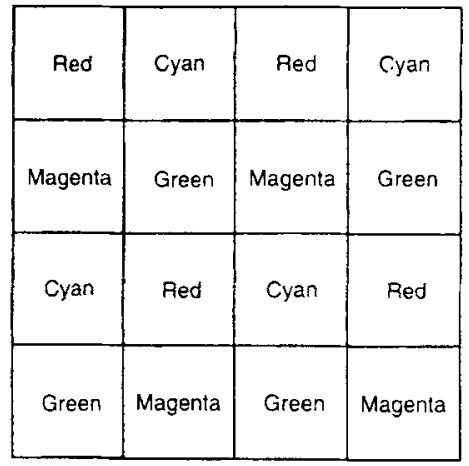

Target : Yellow

Figure 5. Color organization in Experiment 2A. Each small square in the figure is a $2 \times 2$ submatrix of the $8 \times 8$ display matrix. Submatrices in the same column are presented in the same disparity. The figure shows sample displays with the target in yellow and the distractors in the other four colors. In the experiment, the target assumed each of the five colors on an equal number of trials; and for each target color, each submatrix was assigned one of the four distractor colors equally frequently. On half the trials, the row and column arrangements for color and disparity were reversed. Not shown in the figure is the scrambled condition, in which each distractor was randomly assigned a distractor color. 
The average error rate was $2.60 \%$. The correlation between search time and error rate over the experimental conditions was positive and significant $[r(34)=.562$, $p<.05]$. As in Experiment 1, no speed-accuracy tradeoff was evident.

A three-way within-subjects ANOVA was employed to analyze correct search times, with display set size, number of distractor depth planes, and color organization as factors. All main effects were significant: display set size $[F(2,14)=40.47, p<.05]$, number of distractor planes $[F(2,14)=43.04, p<.05]$, and color organization $[F(3,21)=55.08, p<.05]$. The mean search times were $1,178,1,118$, and $1,078 \mathrm{msec}$, for 25,37 , and 49 items, respectively, and they were all significantly different from one another. Search time increased with increases in number of distractor depth planes: $940,1,097$, and 1,336 msec for one, two, and four distractor planes, respectively. Again all these means were significantly different from one another. Finally, search time increased as items of the same color group became less organized. The means for the continuous, small-separation, large-separation, and scrambled conditions of color organization were 992 , $1,082,1,166$, and $1,258 \mathrm{msec}$, respectively. All pairwise differences among these means were significant.

Two two-way interactions were significant. The first was that between number of distractor planes and color organization $[F(6,42)=27.18, p<.05]$. Post hoc analysis showed that search time increased with increases in number of distractor depth planes for all levels of color organization, but the rate of increase was faster when items of the same color became less organized in the $x y$ plane (Figure 6). The second significant interaction was

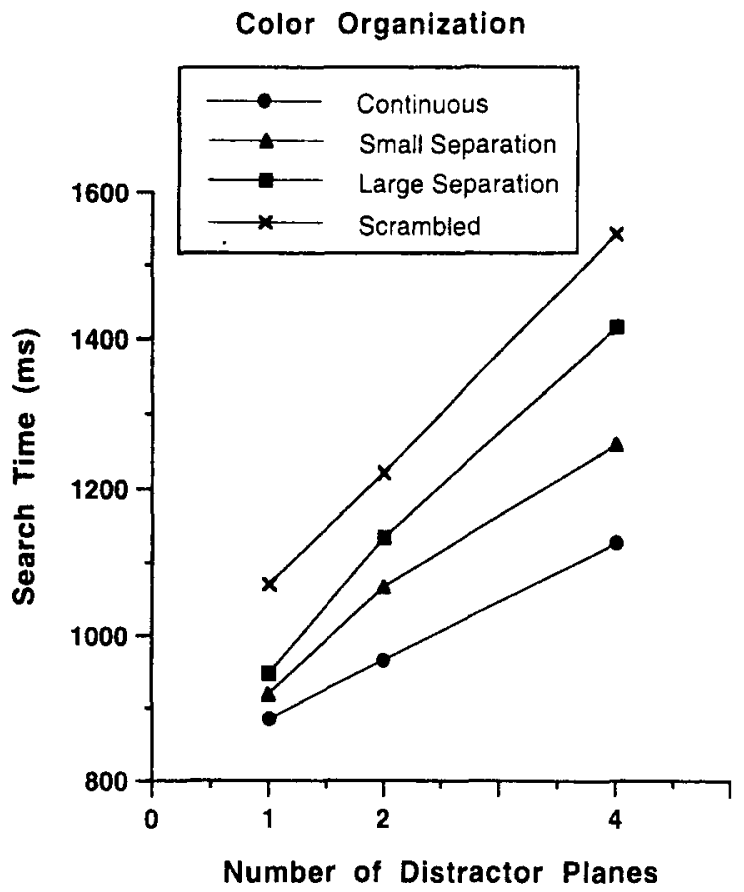

Figure 6. Mean search time as a function of number of distractor planes and color organization in Experiment $2 \mathrm{~A}$. observed between display set size and number of distractor depth planes $[F(4,28)=8.01, p<.05]$. Search time decreased with increases in display set size when there were two or four distractor planes, but remained the same when there was only one distractor plane.

\section{Method: Experiment 2B}

This experiment was similar to Experiment $2 \mathrm{~A}$ except that the manipulation of color organization in Experiment $2 \mathrm{~A}$ was replaced by a similar manipulation of depth plane organization. All five disparities were used on every trial, and distractors of the same color were in spatial contiguity. There were three within-subjects factors: display set size $(25,37$, or 49 items $)$, number of dist ractor colors (one, two, or four), and organization of distractor depth planes. The last factor consisted of four levels and was manipulated in the same manner as was color organization in Experiment $2 \mathrm{~A}$.

\section{Results}

Outlying search times were removed with the same procedure as in the previous experiments. Overall, 1.87 observations (out of 60) were removed from each cell. Only l observer had an average of more than 2 (2.17) observations removed from each cell.

The mean error rate was $2.9 \%$. The correlation between search time and error rate was significant and positive when computed over the 36 experimental conditions $[r(34)=.350, p<.05]$. There was no sign of speedaccuracy tradeoff.

A three-way within-subjects ANOVA was used to analyze the correct search times, with display set size, number of distractor colors, and depth organization as factors. The main effect for number of distractor colors was significant $[F(2,14)=36.90, p<.05]$, as was that for depth organization $[F(3,21)=7.52, p<.05]$. Post hoc comparison showed that the mean search times for one, two, and four distractor colors $(936,1,040$, and $1,244 \mathrm{msec}$, respectively) were significantly different from one another. For depth organization, the mean search times were $1,021,1,074,1,091$, and $1,107 \mathrm{msec}$ for the continuous, small-separation, large-separation, and scrambled conditions, respectively. Responses in the continuous condition were significantly faster than those in the large-separation and scrambled conditions.

Two two-way interactions were significant: number of distractor colors $\times$ depth organization $[F(6,28)=3.22$, $p<.05]$, and display set size $\times$ number of distractor colors $[F(4,28)=10.72, p<.05]$. For the former interaction, search time did not vary with depth organization when there were only one or two distractor colors. In the presence of four distractor colors, observers were faster in the continuous condition than in the other three conditions (Figure 7). The latter interaction was similar to its counterpart in Experiment 1. Search time decreased with increases in display set size only when there were four distractor colors but not when there were one or two distractor colors.

\section{Discussion}

The slower responses to displays with less orderly color or depth organization indicate that it is more diffi- 


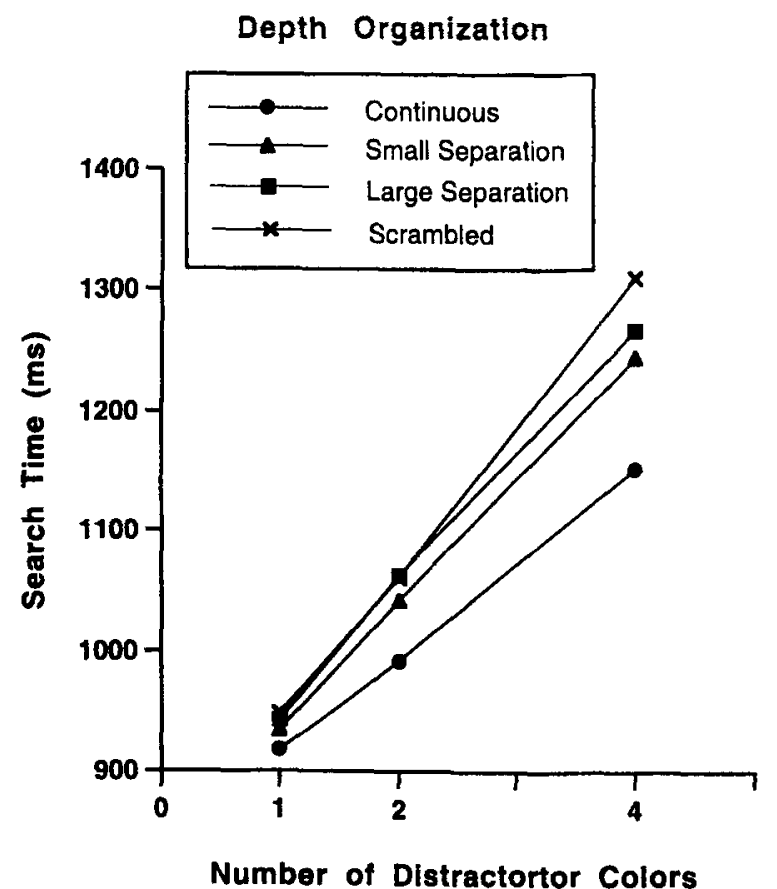

Figure 7. Mean search time as a function of number of distractor colors and depth organization in Experiment 2B.

cult to search for the target when distractors sharing the same attribute are less organized in their $x y$ locations. When distractors of the same color or depth are not presented in spatial proximity, lack of continuation among them and competition from spatially nearby items with different colors or depths render it more difficult to segregate the distractors into the same perceptual unit. As a result, it is more difficult to isolate the target from a background of distractors. The lack of distinct units also makes it more difficult to examine the display by going through the item kernels one by one.

The findings generally support the hypothesis that when organization of items by one dimension becomes weaker, information from the other dimension becomes more important in performing visual search. Figure 6 shows that number of distractor depth planes had a more sizable effect on search time when color organization became less orderly. Though the effect of manipulating depth organization was less notable, the same general trend is seen in Figure 7. These findings are consistent with the notion that observers make optimal use of information according to the effectiveness of the dimensions involved (Ashby \& Maddox, 1990).

Color again appears to be a more useful dimension for identifying the target in disorderly displays. Search time increased as color organization deteriorated, regardless of the number of distractor depth planes (Figure 6). This suggests that color information is always employed to search for the target, even when the target is easily discriminable from the distractors by depth (e.g., the target was an odd item in its own plane when there was only one distractor plane). On the contrary, depth organization had an effect on search time only when there were four distractor colors, but not when there were only one or two distractor colors (Figure 7). In other words, depth information is not very useful in target search when the target is easily distinguishable from the distractors by its color (as in the conditions of one or two distractor colors). Such findings are congruous with Experiment 1, which suggests that color has a stronger effect on search time than stereoscopic depth in visual search in the conditions tested in present experiments.

The interaction between display set size and number of colors and that between display set size and number of distractor planes replicate the corresponding interactions in Experiment 1. Visual search becomes more efficient when additional distractors improve the grouping of distractors.

\section{GENERAL DISCUSSION}

In the present studies, we investigated how information of stereoscopic depth and color is used to perform perceptual segregation and visual search in 3-D visual space. There are five major findings. First, observers use both color and stereoscopic depth information to segregate objects and search for a target in 3-D visual space (Experiment 1). Second, observers search for the target by examining kernels of items sharing the same color and depth (Experiment 1). Third, if one stimulus dimension becomes less effective for distinguishing between the target and the distractors, observers will rely more on the other dimension (Experiment 2). Fourth, target search is sped up by more effective grouping due to increased density of homogeneous distractors (both experiments) or enhanced spatial contiguity among the distractors (Experiment 2). Finally, in comparison with stereoscopic depth, color seems to be a more effective dimension and is always used for object segregation and target search, given the context tested in the present experiments (both experiments).

The first two findings are consistent with the prediction of the holistic processing model, which assumes that selective processing of any visual display depends on the distance among the stimuli in a multidimensional psychological space. As the psychological distance is a composite function of the differences among the stimuli on various dimensions, the model implies that the visual system takes advantage of all relevant information sources to maximize the distinction between the target and distractors so that selective attention can be directed to the relevant stimuli in a speedy and accurate manner. This notion of selective attention being determined by all relevant dimensions is also consistent with the objectbased models (see, e.g., Baylis \& Driver, 1992; Duncan, 1984; Kramer \& Jacobson, 1991; Kramer et al., 1991) and models that emphasize interitem similarity (e.g., Bundesen, 1990; Duncan \& Humphreys, 1989). Such models maintain that items in visual space are parsed into units or groups in the initial preattentive stage ac- 
cording to the principles of perceptual organization, and hence all relevant features have influence on the formation of units and attentional selectivity in visual field. The findings are also in line with the feature inhibition model of Treisman (Treisman, 1988; Treisman \& Sato, 1990), in which attention is directed to the target in the master map through inhibition from all relevant feature maps on locations characterizing the nontarget features. Nevertheless, in relation to the debate of whether color and stereoscopic depth are processed independently, the present conclusion of combined processing of color and depth does not necessarily rule out the possibility that the two dimensions are processed by separate channels. All it suggests is that the outputs of the channels are integrated somewhere in the visual system in performing perceptual segregation and visual search.

The third finding indicates that observers are able to use an optimal rule to combine the two dimensions to search for the target (Ashby \& Maddox, 1990). When one dimension becomes less useful in distinguishing the target from the distractors, they would make up the loss by putting a greater emphasis on the other dimension. Humphreys (1981) has also shown that observers have control over the utility of the different stimulus dimensions and that less attention is allocated to the dimension that specifies the target less reliably.

The fourth finding highlights the importance of display organization in any theory of visual processing. Some current theories of selective attention take into account only the properties of individual items in the display. For instance, the space-based models of attention consider the display only as a collection of items at different localities while neglecting the interactions among the stimulus properties. Such models cannot explain why adding dist ractors (or enhanced grouping among distractors) would reduce search time. This finding, together with the earlier studies that have shown that perceptual organization can strongly affect how selective visual attention is directed (e.g., Kramer \& Jacobson, 1991; Prinzmetal, 1981), suggests that interaction among items in the display is as important as the properties of individual items in determining the formation of perceptual units and direction of selective attention. In this regard, the present findings are more consistent with Duncan and Humphreys's (1989) model than with Treisman's feature inhibition model (Treisman \& Sato, 1990), since the latter does not explicitly state the role of item organization.

In order to develop a comprehensive theory of visual selective attention in 3-D visual space, the effects of spatial and nonspatial factors other than color and stereoscopic depth should be investigated further. For spatial factors, lateral $(x y)$ distance and the separation along the depth axis must be considered (Andersen \& Kramer, 1993). Patterson and Martin (1992) pointed out that lateral separation between stereoscopic stimuli can determine the type (attraction or repulsion) and amount of perceptual interaction among stereoscopic stimuli. For nonspatial factors, the issue is how items in different planes that are similar in the nonspatial dimensions in- teract. Such considerations are necessary to account for the discrepancy between studies that suggest independent processing at different depths (e.g., Gawryszewski, Riggio, Rizzolatti, \& Umiltà, 1987; Nakayama \& Silverman, 1986a, 1986b) and those that suggest an attention gradient along the depth axis (Andersen, 1990; Andersen \& Kramer, 1993). Finally, as shown by the third finding, the relative usefulness of the dimensions involved also affect how different sources of information are utilized in object segregation. All these factors must be considered carefully in mapping out the attentional span in the depth axis of 3-D visual space.

It is unclear why color information had a larger effect on search time than did stereoscopic depth and that it was always used in visual search in our experiments. One possibility is that color is indeed a more powerful dimension than stereoscopic depth for segregation of objects in the preattentive stage because of its higher discriminability. A possible mechanism for this to occur is that distractors sharing the same color exert greater inhibition on one another than do distractors sharing the same depth. Therefore, perceptual units formed from the same color distractors are more differentiable from the one containing the target and hence rejected more readily. Alternatively, it is possible that stereopsis may require more complicated processing computationally because it involves both images from the two eyes and because problems like correspondence have to be solved. Nevertheless, it is also possible that the effect was only due to the specific choice of colors and disparity values in the present experiments. Because color difference and depth separation are measured on different scales, it is hard for us to compare their salience in our study. More research is needed to uncover the cause of this phenomenon.

In conclusion, the present study showed that color and stereoscopic depth information are combined to segregate objects in 3-D visual space. When one dimension becomes less effective in distinguishing the target from the distractors, the other dimension becomes more important. The results support models of visual attention in which selective processing of stimuli is determined by spatial as well as nonspatial stimulus dimensions.

\section{REFERENCES}

Axerstrom, R. A., \& Tond, J. T. (1988). The perception of stereoscopic transparency. Perception \& Psychophysics, 44, 421-432.

ANDERSEN, G. J. (1990). Focused attention in three-dimensional space. Perception \& Psychophysics, 47, 112-120.

Anidersen, G. J., \& Kramer, A. F. (1993). Limits of focused attention in three-dimensional space. Perception \& Psychophysics, 53, 658667.

Anderson, N. H. (1974). Algcbraic models in perception. In E. C. Carterette \& P. M. Friedman (Eds.), Handbook of perception (Vol. 2. pp. 15-98). New York: Academic Press.

AsHBY, F. G., \& MADDOX, W. T. (1990). Integrating information from separable psychological dimensions. Jom of Experimental PS1chology. Human Perception \& Perfornance, 16. 598-612.

BACON, W. F., \& EGETH, H. E. (1991). Local processes in preattentive feature detection. Journal of Experimental Pstcholog.: Human Perception \& Performance, 17, 77-90

Banks, W. P., \& Prinzmeral, W. (1976). Configurational effects in vi- 
sual information processing. Perception \& Psychophysics, 19, 361367.

Baylis, G. C., \& Driver, J. (1992). Visual parsing and response competition: The effect of grouping factors. Perception \& Psychophysics, 51, 145-162.

Beck, J., Prazdny, K., \& Rosenfeld, A. (1983). A theory of textural segmentation. In J. Beck, B. Hope, \& A. Rosenfeld (Eds.), Human and machine vision (pp. 1-38). London: Academic Press.

Bravo, M. J., \& NaKayama, K. (1992). The role of attention in different visual-search tasks. Perception \& Psychophysics, 51, 465-472.

Bundesen, C. (1990). A theory of visual attention. Psychological Review, 97, 523-547.

Bundesen, C., \& Pedersen, L. F. (1983). Color segregation and visual search. Perception \& Psychophysics, 33, 487-493.

Carter, E. C., \& CarTer, R. C. (1981). Color and conspicuousness. Journal of the Optical Society of America, 71, 723-729.

CARTER, R. C. (1982). Visual search with color. Journal of Experimental Psychology: Human Perception \& Performance, 8, 127-136.

Cavanagh, P. (1987). Reconstructing the third dimension: Interactions between color, texture, motion, binocular disparity, and shape. Computer Vision, Graphics \& Image Processing, 37, 171-195.

Coren, S., \& Girgus, J. S. (1980). Principles of perceptual organization and spatial distortion: The Gestalt illusions. Journal of Experimental Psychology: Human Perception \& Performance, 6, 404-412.

DE WEERT, C. M. M. (1979). Color contours and stereopsis. Vision Research, 19, 555-564.

Driver, J., \& Baylis, G. C. (1989). Movement and visual attention: The spotlight metaphor breaks down. Journal of Experimental Psychology: Human Perception \& Performance, 15, 448-456.

Drobnis, B. J., \& Lawson, B. (1976). The Poggendorf illusion in stereoscopic space. Perceptual \& Motor Skills, 42, 15-18.

DUNCAN, J. (1984). Selective attention and the organization of visual information. Journal of Experimental Psychology: General, 113, 501-517.

DunCan, J., \& Humphrevs, G. W. (1989). Visual search and stimulus similarity. Psychological Review, 96, 433-458.

ERIKSEN, B. A., \& ERIKSEN, C. W. (1974). Effects of noise letters upon the identification of a target letter in a nonsearch task. Perception \& Psychophysics, 16, 143-149.

Fox, R., \& Patterson, R. (1981). Depth separation and lateral interference. Perception \& Psychophysics, 30, 513-520.

GarNER, W. R. (1974). Attention: The processing of multiple sources of information. In E. C. Carterette \& P. M. Friedman (Eds.), Handbonk of perception (Vol. 2, pp. 23-59). New York: Academic Press.

Gawryszewski, L. D. G., Riggio, L., Rizzolatti, G., \& Umiltà, C. (1987). Movements of attention in the three dimensions and the meaning of "neutral cues." Neuropsychologia, 25, 19-29.

GREGORY, R. L. (1977). Vision with isoluminant color contrast: 1. A projection technique and observations. Perception, 6, 113-119.

HARMS, L., \& BUndesen, C. (1983). Color segregation and selective attention in a nonsearch task. Perception \& Psychophysics, 33, 11-19.

HuMPhREys, G. W. (1981). Flexibility of attention between stimulus dimensions. Perception \& Psychophysics, 30, 291-302.

Jordan, J. R., III, Geisler, W. S., \& Bovik, A. C. (1990). Color as a source of information in the stereo correspondence process. Vision Research, 30, 1955-1970.

Julesz, B. (1971). Foundations of cyclopean perception. Chicago: University of Chicago Press.
Kramer, A. F., \& JaCOBSON, A. (1991). Perceptual organization and focused attention: The role of objects and proximity in visual processing. Perception \& Psychophysics, 50, 267-284.

Kramer, A. F., Tham, M.-P., \& YeH, Y.-Y. (1991). Movement and focused attention: A failure to replicate. Perception \& Psychophysics, 50, 537-546.

LABERGE, D., \& BRown, V. (1986). Variations in size of the visual field in which targets are presented: An attentional range effect. Perception \& Psychophysics, 40, 188-200.

LeHmkunLe, S., \& Fox, R. (1980). Effect of depth separation on metacontrast masking. Journal of Experimental Psychology: Human Perception \& Performance, 6, 605-621.

Livingstone, M., \& Hubel, D. (1988, May 6). Segregation of form, color, movement, and depth: Anatomy, physiology, and perception. Science, 240, 740-749.

Lu, C., \& Fender, D. H. (1972). The interaction of color and luminance in stereoscopic vision. Investigative Ophthalmology, 11, 482490.

Nakayama, K., \& Silverman, G. H. (1986a). Serial and parallel encoding of feature conjunctions. Investigative Ophthalmology \& Vistual Science, 27 (Suppl.), 182.

Nakayama, K., \& Silverman, G. H. (1986b). Serial and parallel processing of visual feature conjunctions. Nature, 320. 264-265.

Patterson, R., \& Martin, W. L. (1992). Human stereopsis. Human Factors, 34, 669-692.

Prinzmetal, W. (1981). Principles of feature integration in visual perception. Perception \& Psychophysics, 30, 330-340.

SAGI, D., \& Julesz, B. (1987). Short-range limitation on detection of feature differences. Spatial Vision, 2, 39-49.

SCHiller, P. H., Logothetis, N. K., \& Charles, E. R. (1990). Role of color-opponent and broad-band channels in vision. Visual Newoscience, 5, 321-346.

Treisman, A. M. (1988). Features and objects: The Fourteenth Bartlett Memorial Lecture. Quarterly Journal of Experimental Psychology, 40A, 201-237.

Treisman, A. M., \& Sato, S. (1990). Conjunction search revisited. Journal of Experimental Psychology: Human Perception \& Performance, 16, 459-478.

TYler, C. W., \& Cavanagh, P. (1991). Purely chromatic perception of motion in depth: Two eyes as sensitive as one. Perception \& Psychophysics, 49, 53-61.

WAGNer, B., \& BoYnton, R. M. (1972). Comparison of four methods of hetero-chromatic photometry. Journal of the Oprical Society of America, 62, 1508-1515.

YEH, Y.-Y., \& Silverstein, L. (1990). Limits of fusion and depth judgement in stereoscopic display. Human Factors, 32, 45-60.

\section{NOTE}

1. The use of the terms dimension and atribute in this report follows that of Garner (1974). Dimension refers to a stimulus property that varies and serves as an information source. Attribute refers to a single value or level that a stimulus assumes on a dimension.

(Manuscript received October 18. 1994: revision accepted for publication March 3, 1995.) 